\title{
Highly Stable Multicrown Heterostructures of Type-II Nanoplatelets for Ultralow Threshold Optical Gain
}

\author{
Didem Dede, ${ }^{\dagger, \#}$ Nima Taghipour, ${ }^{\dagger, \#}$ Ulviyya Quliyeva, ${ }^{\dagger}$ Mustafa Sak, ${ }^{\dagger}$ Yusuf Kelestemur, ${ }^{\dagger}$ \\ Kivanc Gungor, ${ }^{\dagger}$ and Hilmi Volkan Demir*, ${ }^{\dagger, \ddagger}$
}

${ }^{\dagger}$ Department of Electrical and Electronics Engineering, Department of Physics, UNAM - Institute of Materials Science and Nanotechnology, Bilkent University, Ankara 06800, Turkey

${ }^{\ddagger}$ Luminous! Center of Excellence for Semiconductor Lighting and Displays, School of Electrical and Electronic Engineering, School of Physical and Materials Sciences, School of Materials Science and Nanotechnology, Nanyang Technological University, 639798 Singapore

\section{Supporting Information}

\begin{abstract}
Solution-processed type-II quantum wells exhibit outstanding optical properties, which make them promising candidates for light-generating applications including lasers and LEDs. However, they may suffer from poor colloidal stability under ambient conditions and show strong tendency to assemble into face-to-face stacks. In this work, to resolve the colloidal stability and uncontrolled stacking issues, we proposed and synthesized $\mathrm{CdSe} / \mathrm{CdSe}_{1-x} \mathrm{Te}_{x} / \mathrm{CdS}$ core/multicrown heteronanoplatelets (NPLs), controlling the amount of Te up to $50 \%$ in the crown without changing their thicknesses, which significantly increases their colloidal and photostability under ambient conditions and at the same time preserving their attractive optical properties. Confirming the final lateral growth of CdS sidewalls with $\mathrm{X}$-ray photoelectron spectroscopy, energy-dispersive analysis, and photoelectron excitation spectroscopy, we found that the successful coating of this CdS crown

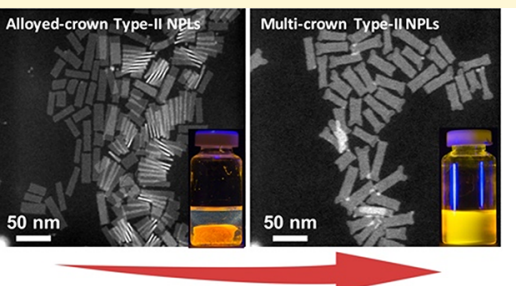

Adapting multi-crown architecture in Type-II NPLS

- Increased colloidal- \& photo-stability

- Decreased scattering (waveguide loss)

- Enhanced optical gain performance around the periphery of conventional type-II NPLs prevents the unwanted formation of needle-like stacks, which results in reduction of the undesired scattering losses in thin-film samples of these NPLs. Owing to highly efficient exciton funneling from the outmost CdS crown accompanied by the reduced scattering and very low waveguide loss coefficient $\left(\sim 18 \mathrm{~cm}^{-1}\right)$, ultralow optical gain thresholds of multicrown type-II NPLs were achieved to be as low as $4.15 \mu \mathrm{J} / \mathrm{cm}^{2}$ and $2.48 \mathrm{~mJ} / \mathrm{cm}^{2}$ under one- and two-photon absorption pumping, respectively. These findings indicate that the strategy of using engineered advanced heterostructures of nanoplatelets provides solutions for improved colloidal stability and enables enhanced photonic performance.
\end{abstract}

\section{INTRODUCTION}

Chemically synthesized semiconductor nanocrystals (NCs) feature strongly size-, shape-, composition-, and structuredependent electronic structures and optical properties. ${ }^{1,2}$ As a result, light-matter interactions in colloidal NCs can be tuned by altering these control parameters, for example, emission spectra and excitonic features. Among these nanocrystal systems, colloidal quantum wells (CQWs), also commonly known as nanoplatelets (NPLs), make a unique class of atomically flat semiconductor NCs thanks to their anisotropic crystal growth facilitating precise control over their thickness. ${ }^{3,4}$ In NPLs, bounded electron-hole pairs (excitons) can freely move in the lateral directions, while they feel strong quantum confinement in the vertical direction. ${ }^{5}$ Thanks to their quasi-2D structure, they possess remarkable excitonic features including strong excitonic peaks in absorption spectra, ${ }^{6}$ large oscillator strength, ${ }^{7}$ ultranarrow emission bandwidth, ${ }^{8}$ and extraordinarily large linear and nonlinear absorption cross sections. ${ }^{8,9}$ All these superior properties of the NPLs make them a versatile and promising material platform for highly efficient nanophotonic applications including light generation (e.g., lasing ${ }^{6,10-12}$ and LEDs $^{13-15}$ ) and light harvesting (e.g., energy transfer ${ }^{16,17}$ and luminescent solar concentrators $\left.^{18}\right)$.

Additionally, advanced heterostructures of the NPLs can be constructed by coating on the top surfaces with laterally and vertically grown shells and also growing on the sidewalls with a peripherally grown crown. ${ }^{19-21}$ By forming core/shell and core/crown heterostructures, one can control the distribution of the electron and hole wave functions, resulting in different types of electronic band alignment including type-I and type-II. In type-I electronic structures, both electron and hole wave functions are confined in the same part (i.e., core) of the heterostructure resulting in large oscillator strength, short photoluminescence (PL) lifetime (a few nanoseconds), and small Stokes shift. ${ }^{19,22,23}$ On the other hand, in type-II heterostructures, due to the recombination of the spatially indirect electrons and holes, the emission profile is strongly

Received: January 11, 2019

Revised: February 10, 2019

Published: February 11, 2019 
red-shifted compared to the absorption spectrum, unlike type-I architectures, which has been previously shown for the CdSe/ $\mathrm{CdTe}$ core/crown NPLs. ${ }^{24-26}$ This material system is relatively new and offers interesting excitonic properties significantly diverging from type-I NPLs. Because the electron and hole wave functions are localized in different parts of the heterostructure, the PL lifetime is longer, and the oscillator strength is lower than those of type-I NPLs. For instance, the Stokes shift reaches $\sim 100 \mathrm{~nm}$, and their lifetime is on the order of a few hundreds of nanoseconds for the $\mathrm{CdSe} / \mathrm{CdTe}$ core/ crown NPLs. $^{24}$

Lately, in addition to the pristine $\mathrm{CdSe} / \mathrm{CdTe}$ core/crown structures, $\mathrm{CdSe} / \mathrm{CdSe} e_{1-x} \mathrm{Te}_{x}$ core/alloyed-crown structures with a precisely controlled crown composition have received great attention. ${ }^{27}$ These engineered heterostructured NPLs provide us with the ability to largely tune excitonic properties, ${ }^{27}$ obtain bicolor emission, ${ }^{28}$ reach high PL quantum efficiency, ${ }^{27}$ and accomplish efficient multiexciton operation. $^{29,30}$ All these properties of $\mathrm{CdSe} / \mathrm{CdSe}_{1-x} \mathrm{Te}_{x}$ core/ alloyed-crown structures make them highly appealing for lightemitting functions. As recently reported by our group, LEDs made from $\mathrm{CdSe} / \mathrm{CdSe} \mathrm{e}_{0.80} \mathrm{Te}_{0.20}$ core/alloyed-crown NPLs show the maximum luminance among the best inverted NPLbased LEDs. ${ }^{31}$ Similarly, in CdSe/CdSe ${ }_{0.50} \mathrm{Te}_{0.50}$ core/alloyedcrown NPLs, the optical gain threshold was achieved to reduce down to $26 \mu \mathrm{J} / \mathrm{cm}^{2}$. 29

Nevertheless, compared to other types of NPL platforms, these $\mathrm{CdSe} / \mathrm{CdSe} e_{1-x} \mathrm{Te}_{x}$ core/alloyed-crown structures are colloidally not stable in solution under ambient conditions, and they also have a strong tendency to form needle-like stacks in their thin-film samples. Even when their surface is coated with a common capping ligand, oleic acid, these NPLs show weak colloidal stability in nonpolar solvents (hexane, toluene, etc.), forming aggregates soon after their synthesis. In the literature, there is no report specifically about the colloidal stability of $\mathrm{CdSe} / \mathrm{CdSe}_{1-x} \mathrm{Te}_{x}$ NPLs in solution and no previous work that addresses the issues of their colloidal instability and undesired stacking, to the best of our knowledge.

Herein, to address the colloidal stability under ambient conditions, we designed and synthesized multicrown NPLs by growing a $\mathrm{CdS}$ crown around the periphery of $\mathrm{CdSe} /$ $\mathrm{CdSe}_{1-x} \mathrm{Te}_{x}$ core/alloyed-crown structures. We performed the proposed CdS growth systematically for different alloyedcrown compositions, which especially worked well in the crown systems having a Te composition less than $50 \%$. We observed that the $\mathrm{CdS}$ periphery coating substantially increased their colloidal stability in nonpolar solvents, while there was no significant change observed in the emission spectra except for a few nanometer red shift as compared to the noncoated samples. With increased stability, we also systematically studied the one- and two-photon absorption (1PA and 2PA)-pumped optical gain performances of these $\mathrm{CdSe} / \mathrm{CdSe}_{1-x} \mathrm{Te}_{x} / \mathrm{CdS}$ multicrown NPLs having different $\mathrm{Te}$ compositions to understand the effect of $\mathrm{CdS}$ sidewalls. We found that the prosperous growth of $\mathrm{CdS}$ crowns allows for efficient exciton funneling from the added CdS wings to the starting core/alloyed-crown region and significant reduction in the optical waveguide loss coefficient in the closely packed films of these NPLs, which enables ultralow amplified spontaneous emission (ASE) thresholds. As a result, we achieved 1PA- and 2PA-pumped ASE thresholds for $x=0.25$ as low as $4.15 \mu \mathrm{J} / \mathrm{cm}^{2}$ and $2.48 \mathrm{~mJ} / \mathrm{cm}^{2}$, respectively. To the best of our knowledge, these attained ASE thresholds are the best in their class among all heterostructures of the NPLs and QDs. Therefore, the strategy of CdS crown coating around the periphery of such alloyed-crown type-II NPLs not only makes them a highly stable colloidal system but also an excellent candidate for gain medium in lasing applications.

\section{EXPERIMENTAL SECTION}

Chemicals. Cadmium nitrate tetrahydrate $\left[\mathrm{Cd}\left(\mathrm{NO}_{3}\right)_{2} \cdot 4 \mathrm{H}_{2} \mathrm{O}\right]$ (99.999\% trace metal basis), cadmium acetate dihydrate [Cd$\left.(\mathrm{OAc})_{2} \cdot 2 \mathrm{H}_{2} \mathrm{O}\right](>98 \%)$, sodium myristate $(>99 \%)$, technical-grade 1-octadecene (ODE), tellurium (Te), selenium (Se), sulfur (S) (99.998\% all are trace metals basis), technical-grade oleic acid (OA) (90\%), trioctylphoshine (TOP), hexane, ethanol, methanol, toluene, were purchased from Sigma Aldrich.

Preparation of Cadmium Myristate. Cadmium myristate was synthesized according to the previously published recipe in the literature. ${ }^{19}$ In a typical synthesis, $2.46 \mathrm{~g}$ of $\mathrm{Cd}\left(\mathrm{NO}_{3}\right)_{2} \cdot 4 \mathrm{H}_{2} \mathrm{O}$ was dissolved in $80 \mathrm{~mL}$ of methanol, and $6.26 \mathrm{~g}$ of sodium myristate was dissolved in $500 \mathrm{~mL}$ of methanol by continuous stirring. After complete dissolution, both solutions were mixed, and stirring was continued for $\sim 2 \mathrm{~h}$. Then, white bulky precipitates of cadmium myristate were washed and cleaned with methanol. Finally, the precipitate was dried under vacuum for approximately $24 \mathrm{~h}$.

Synthesis of Four-ML-Thick CdSe NPLs. For the synthesis of four-ML-thick CdSe core NPLs, $340 \mathrm{mg}$ of cadmium myristate, $24 \mathrm{mg}$ Se and $30 \mathrm{~mL}$ of ODE were added in a $100 \mathrm{~mL}$ three-neck flask and the solution was degassed under vacuum at $90{ }^{\circ} \mathrm{C}$ around $1 \mathrm{~h}$. Then, the temperature of the solution was set to $240{ }^{\circ} \mathrm{C}$ under argon (Ar) flow. When the temperature reached $\sim 185{ }^{\circ} \mathrm{C}, 90 \mathrm{mg}$ of $\mathrm{Cd}(\mathrm{OAc})_{2}$. $2 \mathrm{H}_{2} \mathrm{O}$ was added. After $9 \mathrm{~min}$ growth at $240{ }^{\circ} \mathrm{C}, 1 \mathrm{~mL}$ of OA was injected and the solution was moderately cooled to room temperature. Below $120{ }^{\circ} \mathrm{C}, 5 \mathrm{~mL}$ of hexane was injected for better dissolution of NPLs. In the purification stage NPLs were precipitated by adding ethanol and then kept in hexane solution.

Synthesis of Four-ML-Thick CdSe/CdSe ${ }_{1-x} \mathrm{Te}_{x}$ Core/AlloyedCrown NPLs. For the laterally grown alloyed crown, a similar method in the literature was used. ${ }^{27,28}$ In a $25 \mathrm{~mL}$ flask, $1.5 \mathrm{~mL}$ of CdSe NPL solution, which was cleaned once with ethanol and dissolved in $4 \mathrm{~mL}$ of ODE, $30 \mathrm{mg}$ of $\mathrm{Cd}(\mathrm{OAc})_{2} \cdot 2 \mathrm{H}_{2} \mathrm{O}$, and $45 \mu \mathrm{L}$ of OA were mixed and slowly degassed at room temperature (RT) for approximately 30 $\mathrm{min}$. Then, under an inert atmosphere, the temperature was raised to $215^{\circ} \mathrm{C}$, and the mixtures of TOP-Se, TOP-Te, and ODE (0.03 M) were injected at $2 \mathrm{~mL} / \mathrm{h}$. (TOP-Se and TOP-Te precursors having a concentration of $1 \mathrm{M}$ were prepared inside a glovebox.) After the injection, the solution was maintained at the same temperature for 1 min, and the reaction was stopped by the addition of $0.5 \mathrm{~mL}$ of OA. Then, the mixture was cooled down to $\mathrm{RT}$, and $5 \mathrm{~mL}$ hexane was added for better dissolution and kept as-synthesized for CdS growing.

Preparation of Anisotropic Growth Solution for Four-MLThick CdS Crown Region. For the lateral growth of the CdS crown, $\mathrm{Cd}$ and $\mathrm{S}$ precursors were prepared according to the well-known procedure with slight modifications. ${ }^{19} \mathrm{Cd}(\mathrm{OAc})_{2} \cdot 2 \mathrm{H}_{2} \mathrm{O}(960 \mathrm{mg})$, $680 \mu \mathrm{L}$ of $\mathrm{OA}$, and $4 \mathrm{~mL}$ of $\mathrm{ODE}$ were stirred at $120{ }^{\circ} \mathrm{C}$ under an ambient atmosphere and regularly sonicated. Alternating steps of heating and sonication followed until a whitish homogeneous gel formed. As a sulfur source, $0.15 \mathrm{M} \mathrm{S}$ in ODE was prepared at $125^{\circ} \mathrm{C}$ under ambient conditions. Right before injection, precursors were mixed at a 1:1 ratio.

Synthesis of Four-ML-Thick $\mathrm{CdSe} / \mathrm{CdSe}_{1-x} \mathrm{Te}_{x} / \mathrm{CdS}$ Core/ Alloyed-Crown/Crown NPLs. To grow the CdS around the periphery of type-II NPLs, an anisotropic growth mixture was prepared. Type-II as-synthesized solution $(8 \mathrm{~mL})$ was cleaned with ethanol and dissolved in $0.4 \mathrm{~mL}$ of hexane and $4 \mathrm{~mL}$ of ODE. Then, the solution is placed in a $25 \mathrm{~mL}$ flask together with $50 \mu \mathrm{L}$ of OA and degassed at RT. Under Ar flow, the temperature was set to $225^{\circ} \mathrm{C}$, and at $180^{\circ} \mathrm{C}$, injection of the $\mathrm{Cd}-\mathrm{S}$ precursor mixture was started. Right after the required amount of precursor was injected, the reaction was finalized by water cooling. Then, $5 \mathrm{~mL}$ of hexane was added, and the resulting solution was centrifuged at $3000 \mathrm{rpm}$ for 3 
min. Normally, after CdS coating, there was almost no precipitate. Finally, the supernatant was washed with ethanol at $6000 \mathrm{rpm}$ for $\sim 6$ min and stored in toluene.

Absorption, Steady-State Photoluminescence (PL), and Photoexcitation (PLE) Spectroscopy. UV-vis absorption spectra of NPL samples were taken by using Cary $100 \mathrm{UV}-\mathrm{vis}$, and PL and PLE spectra were taken by using a Cary Eclipse fluorescence spectrophotometer. Samples were dissolved in toluene.

Photoluminescence Quantum Yield (PL-QY) and Stability Measurements. Our setup for PL-QY and stability measurements was equipped with an Ocean Optics Maya 2000 spectrometer, an integrating sphere, a xenon lamp, and $\alpha$ monochromator. The methodology used for PL-QY was described by de Mello et al., ${ }^{32}$ and the excitation wavelength was $375 \mathrm{~nm}$. For stability measurements, dispersions of NPLs having an optical density of $\sim 1$ (at the first excitonic peak of CdSe, $\sim 513 \mathrm{~nm}$ ) were excited also at a wavelength of $375 \mathrm{~nm}$, and their spectra were recorded at different time intervals for $24 \mathrm{~h}$. Between the time intervals, solutions were kept in the dark.

Time-Resolved Photoluminescence Spectroscopy. The timeresolved photoluminescence measurements were taken by using a PicoQuant FluoTime 200 spectrometer. Cuvettes filled with NPLs dispersions were excited with a picosecond pulsed laser having a wavelength of $375 \mathrm{~nm}$. Then, the fluorescence decay curves were recorded with a TimeHarp time-correlated single-photon counting (TCSPC) unit.

Transmission Electron Microscopy. High-angle annular darkfield scanning transmission electron microscopy (HAADF-STEM) images of diluted solutions of NPLs were acquired with FEI Tecnai G2 F30 which was operated at $300 \mathrm{kV}$.

$\mathrm{X}$-ray Photoelectron Spectroscopy. For the XPS measurements, a Thermo Scientific K $\alpha \mathrm{X}$-ray photoelectron spectrometer was used. The ratio between $\mathrm{Se}$ and $\mathrm{Te}$ was determined for $\mathrm{CdSe} /$ $\mathrm{CdSe}_{1-x} \mathrm{Te}_{x}$ NPLs, and then, the presence of $S$ was shown in their multicrown counterparts.

\section{RESULTS AND DISCUSSION}

In this study, we first investigated the reasons that lead to instability of the core/alloyed-crown type-II NPLs in solution under ambient conditions. To synthesize core/alloyed-crown type-II NPLs, we started with four-monolayer (ML)-thick CdSe core NPLs. These core NPLs have a PL emission peak centered at $513 \mathrm{~nm}$ with a FWHM of $\sim 10 \mathrm{~nm}$, (see the Supporting Information, Figure S1). Afterward, CdSe/ $\mathrm{CdSe}_{1-x} \mathrm{Te}_{x}$ core/alloyed-crown type-II NPLs with six different compositions (for $x=0.05,0.10,0.25,0.40,0.50$, and 1.00) were synthesized according to our previously reported procedure with slight modifications. ${ }^{27}$ To confirm successful syntheses, the compositions of the resulting core/alloyedcrown type-II NPLs were determined by X-ray photoelectron spectroscopy (XPS). The final Se and Te compositions of the alloyed crown (Table S1) were found to be well-controlled by the injected precursor amounts. We kept the core/alloyedcrown type-II NPLs both dissolved in a nonpolar solvent (toluene) and as-synthesized. In the latter case, NPLs were observed to rearrange themselves to form gel-like structures in the presence of 1-octadecene (ODE) as shown in Figure 1a.

To observe this structure of the resultant self-composed system, gel-like samples were imaged using high-angle annular dark-field scanning transmission electron microscopy (HAADF-STEM). As can be seen in Figure 1b, NPLs form face-to-face stacks, and this structure resembles chiral ribbons of five-ML CdSe NPLs, which were previously reported to be formed by adding excess oleic acid with the slow evaporation of nonpolar solvent. ${ }^{33}$ ODE $\left(\mathrm{C}_{18} \mathrm{H}_{36}\right)$ might have caused the same ribbon conglomeration of our core/alloyed-crown NPLs due to its structural similarity to oleic acid $\left(\mathrm{C}_{18} \mathrm{H}_{34} \mathrm{O}_{2}\right)$. (a)

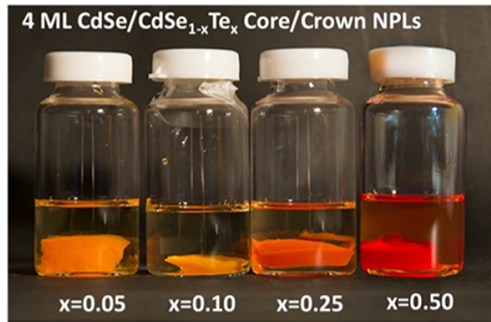

(b)

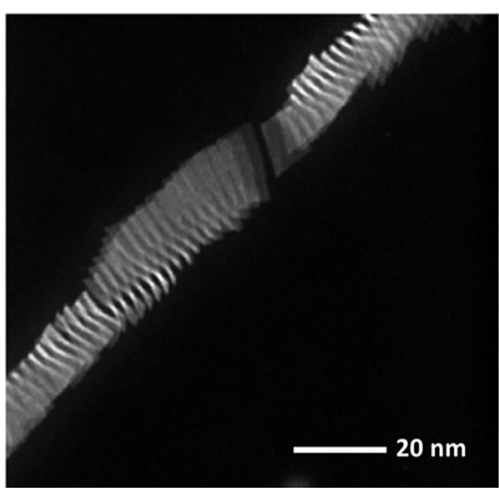

Figure 1. (a) Real color images of $\mathrm{CdSe} / \mathrm{CdSe}_{1-x} \mathrm{Te}_{x}$ core/alloyedcrown type-II NPLs having different alloyed-crown compositions kept as-synthesized under ambient conditions. (b) High-angle annular dark-field scanning transmission electron microscopy (HAADFTEM) image of gel-like $\mathrm{CdSe} / \mathrm{CdSe}_{0.50} \mathrm{Te}_{0.50}$ core/alloyed-crown type-II NPLs.

However, in these type-II NPLs dissolved even in the nonpolar solvent, precipitation was found to commence in a few days, and characteristic needle-like stacking occurred in their spincoated thin films. ${ }^{28,29,34}$ Although this does not completely exclude the role of ODE in the formation of a gel-like structure, the main reason for stacking may actually lie in the structure of our core/alloyed-crown NPLs.

As investigated previously by Abećassis et al., only the NPLs having well-defined edges self-assemble to form superlattices. ${ }^{33}$ Thus, the highly uniform shape of type-II NPLs exhibiting more razor-sharp edges as compared to type-I NPLs (i.e., $\mathrm{CdSe} / \mathrm{CdS}$ core/crown) may trigger colloidal instability or a tendency to pile up in face-to-face stacks. In addition, stacking could also possibly be facilitated by the type-II electronic band alignment of these core/alloyed-crown NPLs. In type-II NPLs, the partial separation of electron-hole pairs may cause localized charge distribution over the structure generating strong attractions in addition to van der Waals interactions between nearby NPLs. ${ }^{29}$ Finally, Te is oxidized more easily than $\mathrm{S}$ or Se, which might contribute to the decreased stability in these materials. A systematic set of detailed experiments is needed to study these effects.

Here, to look into these issues, we employed a multicrown architecture by additionally growing a CdS crown around the periphery of the starting type-II heterostructure, and for the synthesis of the resulting $\mathrm{CdSe} / \mathrm{CdSe} e_{1-x} \mathrm{Te}_{x} / \mathrm{CdS}$ NPLs, we modified the recipe of four-ML-thick $\mathrm{CdSe} / \mathrm{CdS}$ core/crown NPLs. The addition of $\mathrm{Cd}$ and $\mathrm{S}$ precursors results in anisotropic growth of $\mathrm{CdS}$ around the corners of the rectangular-shaped type-II seeds. The presence of sulfur was verified by the Auger LMM peak of sulfur by XPS and also energy-dispersive analysis inside TEM (given in Figures S5 and S6, respectively). The HAADF-TEM images of four-ML-thick 
(a)

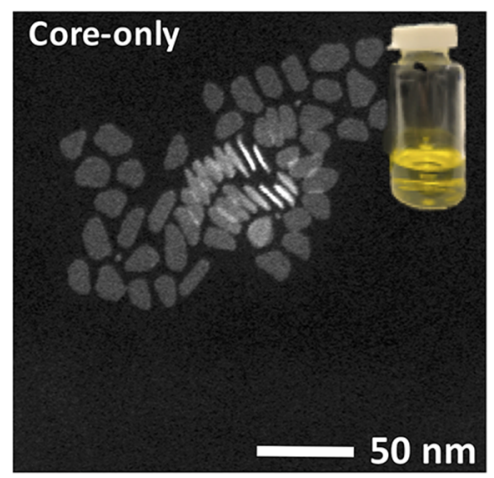

(b)

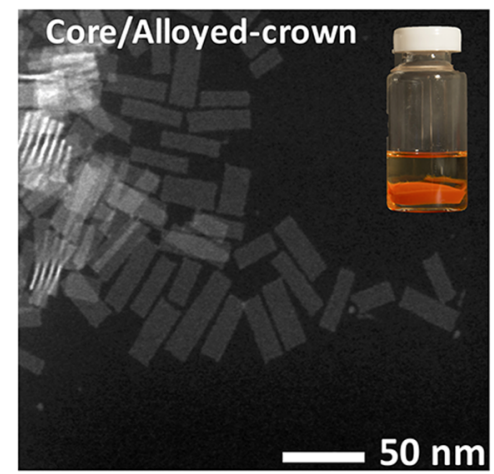

(c)

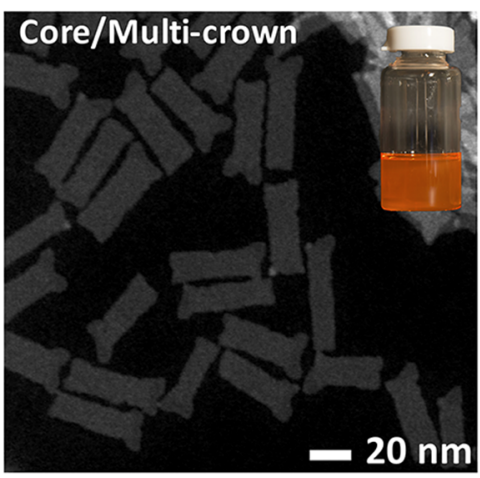

(d)

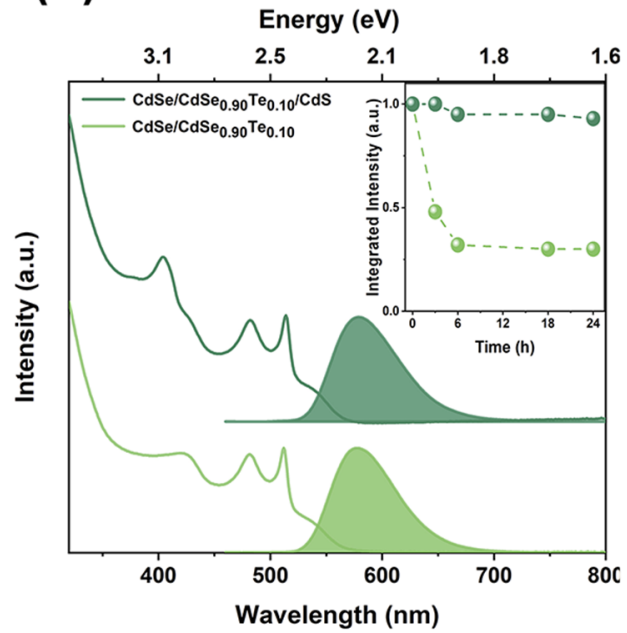

(e)

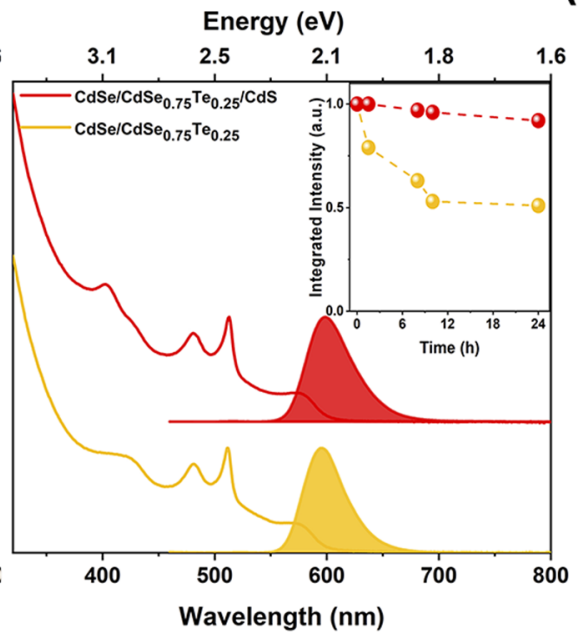

(f)

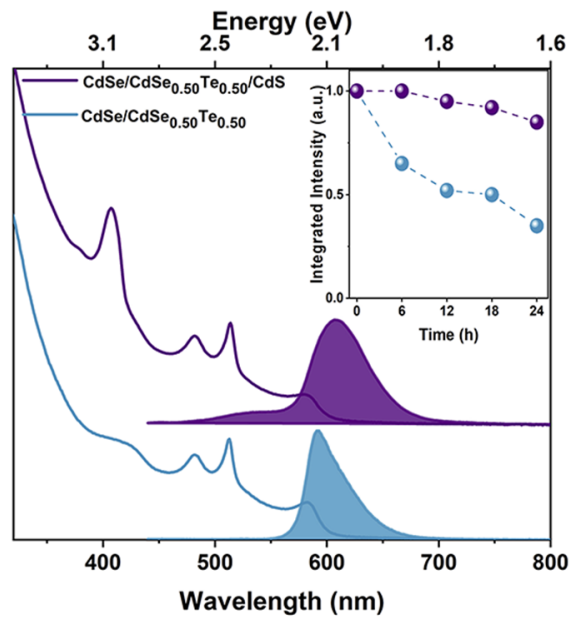

Figure 2. HAADF-STEM images of (a) four-ML CdSe core-only, (b) CdSe/CdSe ${ }_{0.75} \mathrm{Te}_{0.25}$ core/alloyed-crown, and (c) CdSe/CdSe ${ }_{0.75} \mathrm{Te}_{0.25} / \mathrm{CdS}$ core/alloyed-crown/crown NPLs. Steady-state photoluminescence and absorption spectra of $\mathrm{CdSe} / \mathrm{CdSe}_{1-x} \mathrm{Te}_{x}$ core/alloyed-crown and CdSe/ $\mathrm{CdSe}_{1-x} \mathrm{Te}_{x} / \mathrm{CdS}$ core/alloyed-crown/crown are given for the alloyed-crown compositions of (d) $x=0.10$, (e) 0.25 , and (f) 0.50 . Inset figures show the PL stability tests conducted for each sample.

CdSe core-only, CdSe/CdSe $e_{0.75} \mathrm{Te}_{0.25}$ core/alloyed-crown, and $\mathrm{CdSe} / \mathrm{CdSe}_{0.75} \mathrm{Te}_{0.25} / \mathrm{CdS}$ multicrown NPLs can be seen in Figure $2 \mathrm{a}-\mathrm{c}$, respectively. Although the bare core NPLs exhibit nonuniform shapes, by growing $\mathrm{CdSe}_{1-x} \mathrm{Te}_{x}$ alloyed-crown, the shape of the structure becomes highly uniform with extremely sharp edges and corners. As seen in Figure 2c, after the growth of a CdS crown, the rectangular shape of the type-II NPLs became irregular. The uniform $\mathrm{CdS}$ growth is challenging also in $\mathrm{CdSe} / \mathrm{CdS}$ core/crown type-I NPLs. In our case, nonuniformity of the shape may be one reason for enhancing the colloidal stability of type-II NPLs. The destruction of their perfect symmetrical shapes by $\mathrm{CdS}$ crown growth significantly diminishes the probability of NPL stacking. Images for the other compositions of core/alloyed-crown and their $\mathrm{CdS}$ coated crown are also shown in Figure S2.

The structural characterizations of the NPLs were followed by the steady-state optical characterizations including UV-vis absorption, photoluminescence (PL), and photoluminescence excitation (PLE) spectroscopy. Although the core-only NPLs exhibit a narrow emission peaking at $513 \mathrm{~nm}$ (Figure S1), the $\mathrm{CdSe} / \mathrm{CdSe} \mathrm{1}_{1-x} \mathrm{Te}_{x}$ core/alloyed-crown NPLs show strongly red-shifted PL emissions due to the recombination of the electron-hole pair at the charge-transfer state. Depending on the ratio between $\mathrm{Se}$ and $\mathrm{Te}$ in the crown region, $\mathrm{PL}$ emission shifts from pure CdSe to the CdTe crown. In Figure $2 \mathrm{~d}-\mathrm{f}$, absorption and emission spectra of the core/alloyed-crown and multicrown samples can be seen for the compositions of $\mathrm{x}=$ $0.10,0.25$, and 0.50 (see also Figure S3) As presented in Figure $2 \mathrm{~d}, \mathrm{e}, \mathrm{CdS}$ growth around the periphery causes a slight red shift in the PL emission ( $\sim 2$ to $3 \mathrm{~nm})$. In Figure $2 \mathrm{f}$, there is a tail formation at higher photon energies as a result of the separate CdS nucleation, which was also confirmed by PLE spectroscopy (Figure S5). We attribute this behavior to the lattice mismatch between $\mathrm{CdSe}_{1-x} \mathrm{Te}_{x}$ and $\mathrm{CdS}$, which is directly proportional to the amount of Te incorporated in the alloyed crown. This makes the uniform growth of $\mathrm{CdS}$ around the periphery of core/alloyed-crown NPLs extremely difficult for higher amount of $\mathrm{Te}(x \geq 0.50)$ in the core/alloyed-crown structure. We also verified the separate nucleation of $\mathrm{CdS}$ by growing around the periphery of the pure $\mathrm{CdSe} / \mathrm{CdTe}$ core/ crown sample (Figure S7). Insets of Figure $2 \mathrm{~d}-\mathrm{f}$ show the photostability tests performed for the samples in solution, all having the same concentration. Highly concentrated samples were kept in cuvettes and carefully handled. The PL measurements were taken in our setup in time periods of 24 $\mathrm{h}$, and integrated photon intensity was recorded as a function of time. It can be seen that in shorter than $12 \mathrm{~h}$, the integrated intensity of the core/alloyed-crown type-II samples decreased 
(a)

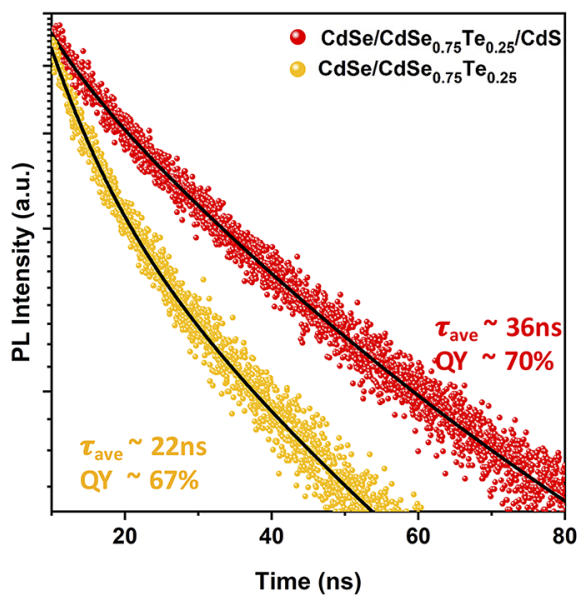

(b)

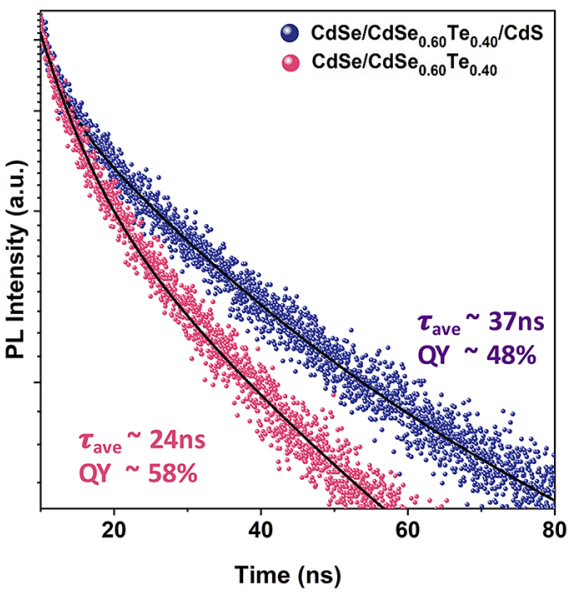

(c)

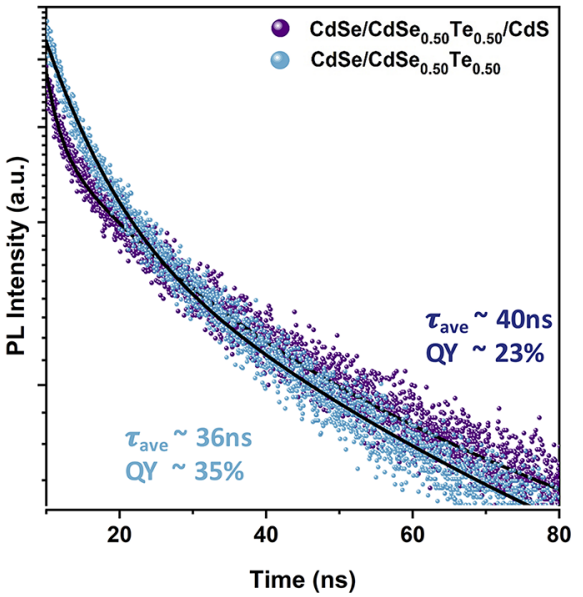

Figure 3. Time-resolved fluorescence decay curves and fittings of $\mathrm{CdSe} / \mathrm{CdSe}_{1-x} \mathrm{Te}_{x}$ core/alloyed-crown and CdSe/CdSe $e_{1-x} \mathrm{Te}_{x} / \mathrm{CdS}$ multicrown samples having Te compositions of (a) $\mathrm{x}=0.25$, (b) 0.40 , and (c) 0.50 . The amplitude-averaged lifetime of the samples and their $\mathrm{QY}$ are provided next to the respective decay curves in the figures.
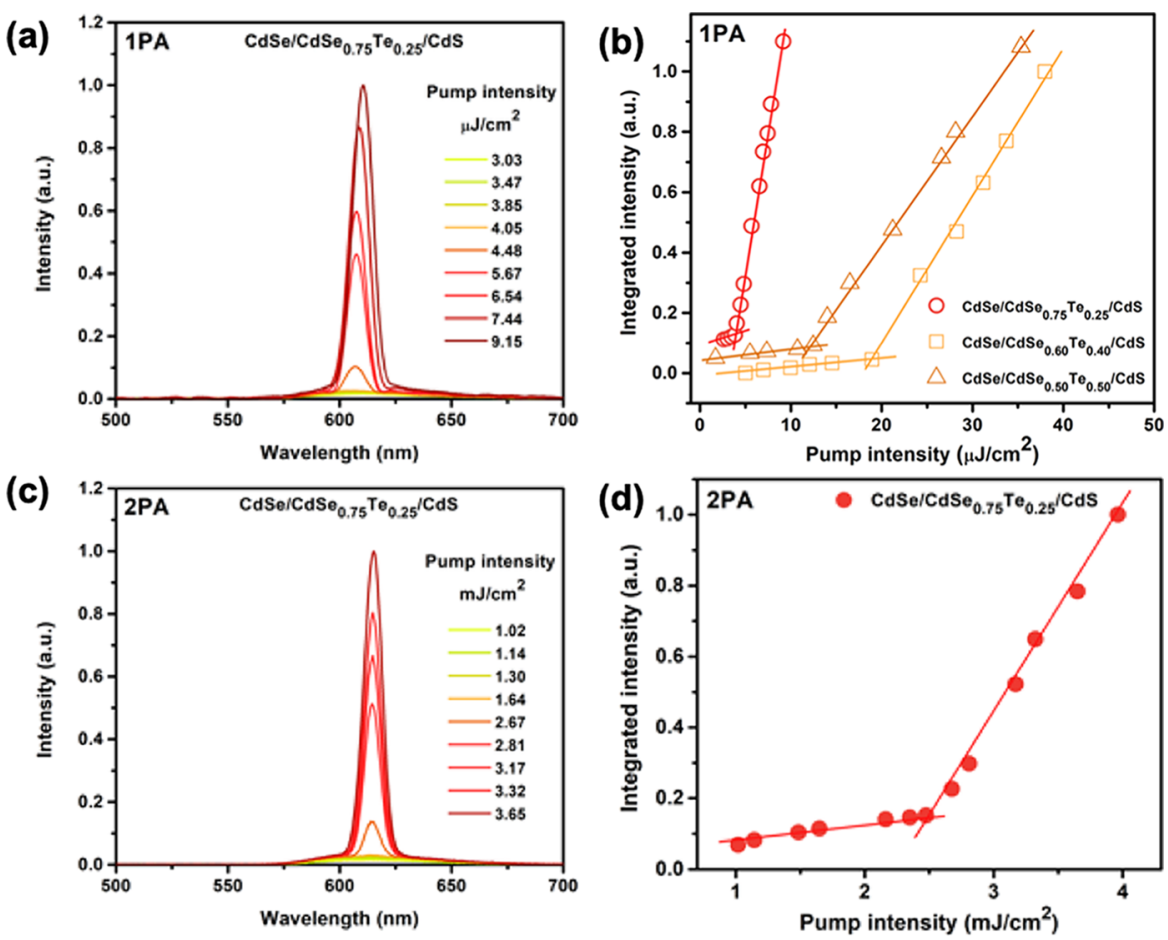

Figure 4. (a) Photoluminescence spectra of the multicrown CdSe/CdSe $e_{0.75} \mathrm{Te}_{0.25} / \mathrm{CdS}$ type-II NPLs under single-photon absorption excitation $\left(\lambda_{\text {exc }}=400 \mathrm{~nm}\right)$. (b) Integrated PL intensity as a function of pump intensity under 1PA excitation. (c) PL spectra of the multicrown CdSe/ $\mathrm{CdSe}_{0.75} \mathrm{Te}_{0.25} / \mathrm{CdS}$ type-II NPLs under two-photon absorption excitation $\left(\lambda_{\text {exc }}=800 \mathrm{~nm}\right.$ ). (d) Integrated PL intensity as a function of pump intensity under 2PA excitation.

at least by $60 \%$, where this decrease is smaller than $10 \%$ for the multicrown structures regardless of their crown composition, showing the decreased colloidal stability with respect to time.

Furthermore, to understand the photophysical behavior of our heteronanoplatelets, we performed absolute PL quantum yield (QY) and time-resolved fluorescence (TRF) spectroscopy measurements. To analyze the fluorescence decay kinetics of the samples, the PL decay curves were fitted by multiexponential functions, and the results are summarized in Table S2 (Supporting Information) together with their PLQYs. In Figure 3a-c, fluorescence decay curves of the core/ alloyed-crown and multicrown samples are presented for different amounts of $\mathrm{Te}(x=0.25,0.40$, and 0.50$)$ together with their amplitude-averaged lifetimes and PL-QYs. As can be seen, there is an increase in the PL-QY of the multicrown samples up to the low amount of $\mathrm{Te}(x \leq 0.25)$, whereas there is a decrease for higher Te amounts $(x>0.25)$ in the alloyedcrown region. We ascribe this behavior to the increased lattice mismatch between CdTe and CdS in the multicrown NPLs. The bulk CdTe has a lattice constant of $6.4 \AA$, whereas this is $5.8 \AA$ for $\mathrm{CdS}$ in their cubic lattice structures. ${ }^{35}$ This larger lattice mismatch might make it more difficult to grow CdS 

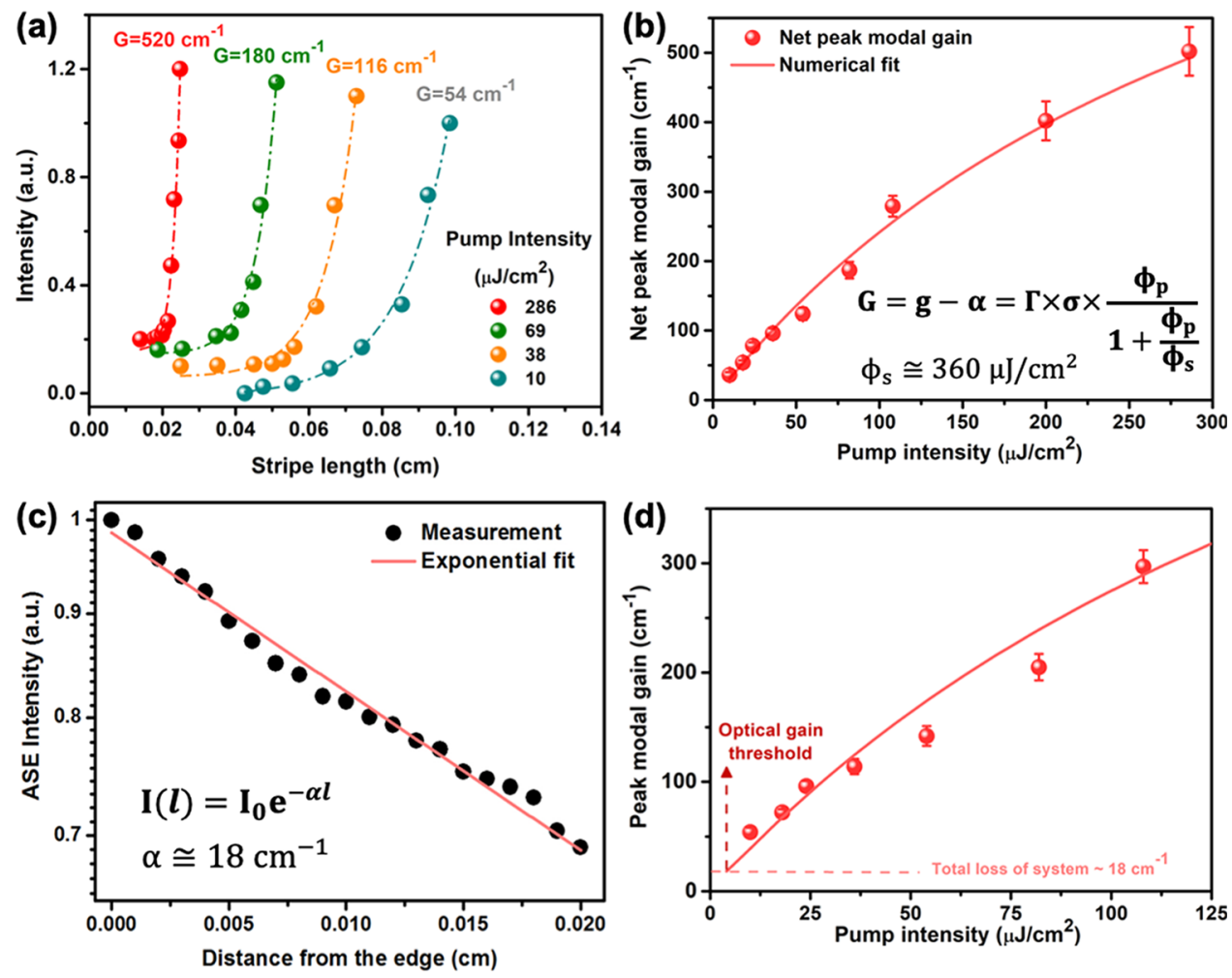

Figure 5. (a) Variable stripe length (VSL) measurements of the multicrown CdSe/CdSe ${ }_{0.75} \mathrm{Te}_{0.25} / \mathrm{CdS}$ type-II NPLs parameterized with respect to the pumping intensity. Here, the dash-dotted lines show the fitting curves of the VSL data by using eq 1. (b) Net peak modal gain ( $G$ ) as a function of the pump fluence fitted by eq 2 . where the highest $G$ reaches $520 \mathrm{~cm}^{-1}$ at $\sim 286 \mu \mathrm{J} / \mathrm{cm}^{2}$ pump intensity, while the saturation pump intensity $\left(\phi_{\mathrm{s}}\right)$ is $\sim 360 \mu \mathrm{J} / \mathrm{cm}^{2}$. (c) Total loss of system (waveguide loss coefficient) measurement $(\alpha)$. (d) Peak modal gain $(g)$ as a function of pump intensity where the total system loss is $\sim 18 \mathrm{~cm}^{-1}$. The optical gain occurs when the modal gain equals to the total loss of system $(g=\alpha)$.

around the periphery and may thus promote the separate nucleation of CdS NCs and/or NPLs. Moreover, the PL lifetimes of the multicrown samples were increased compared to their core/alloyed-crown type-II counterparts. As seen previously in $\mathrm{CdSe} / \mathrm{CdS}$ type-I core/crown NPLs, this elongation of the PL decay curves in the multicrown structure can be explained with the improved sidewall passivation thanks to the CdS coating around the periphery.

Next, we have investigated the optical gain performance of these highly stable multicrown $\mathrm{CdSe} / \mathrm{CdSe}_{1-x} \mathrm{Te}_{x} / \mathrm{CdS}$ type-II NPLs. To do so, we prepared closely packed thin films of the developed NPLs via spin-coating and drop-casting techniques. According to our observations, the spin-coated thin-film samples (thickness of $\sim 180 \pm 20 \mathrm{~nm}$ ) exhibited lower ASE thresholds than the drop-casted ones. This can be attributed to the reduced optical waveguide losses thanks to the improved film formation in the spin-coated samples of these NPLs. We then performed the optical gain measurements using spincoated samples. To minimize the degradation of the samples under ASE measurements and protect the samples from oxygen and moisture, we encapsulated the NPL films in a nitrogen-filled glovebox by using epoxy (Figure S8). To study the ASE of these samples, we pumped them under stripe excitation of a femtosecond mode-locked Ti:sapphire regenerative amplifier (Spitfire Pro, Spectra Physics) laser having $120 \mathrm{fs}$ pulse width and $1 \mathrm{kHz}$ repetition rate operating at 800 $\mathrm{nm}$ (or $\sim 400 \mathrm{~nm}$ in frequency-doubled using a BBO crystal).

Photoluminescence spectra of the multicrown $\mathrm{CdSe} /$ $\mathrm{CdSe}_{0.75} \mathrm{Te}_{0.25} / \mathrm{CdS}$ samples under single-photon absorption excitation $\left(\lambda_{\text {exc }}=400 \mathrm{~nm}\right)$ are shown as an exemplary case in
Figure 4a (see Figure S9 for other samples). The transition from the spontaneous emission to the ASE is clearly seen in Figure $4 \mathrm{a}$ where an abrupt rise in the integrated intensity and narrowing in the full width at half maximum (FWHM) of $\sim 9$ $\mathrm{nm}$ are observed. In multicrown $\mathrm{CdSe} / \mathrm{CdSe}{ }_{0.75} \mathrm{Te}_{0.25} / \mathrm{CdS}$ type-II NPLs, the spontaneous emission and ASE peaks are centered at 613 and $610 \mathrm{~nm}$, respectively. Thus, the ASE is slightly blue-shifted $(\sim 3 \mathrm{~nm})$ compared to the spontaneous emission. Here, the origin of the blue-shifted ASE is not due to the repulsive exciton-exciton interaction, which has been generally reported for QDs having type-II electronic structures. In these NPLs, the ASE stems from the exciton localized in the crown region coupled to the charge-transfer state. ${ }^{29,30,36,37}$

Here, by growth of the $\mathrm{CdS}$ crown in the periphery of alloyed-crown CdSe/CdSe ${ }_{1-x} \mathrm{Te}_{x} \mathrm{NPLs}$, the first excitonic peak of the four-ML CdS crown appears at $407 \mathrm{~nm}$ (Figure 2), which also emerges in the PLE spectra (Figure S4). As a consequence, the photogenerated excitons in the $\mathrm{CdS}$ crown were rapidly transferred to the $\mathrm{CdSe} / \mathrm{CdSe} e_{1-x} \mathrm{Te}_{x}$ core/alloyed crown via exciton funneling, which was previously reported in the conventional core/crown NPLs, core/shell QDs, and nanorods. ${ }^{38}$ Therefore, in our core/alloyed-crown/crown heteronanoplatelets, the optical absorption is significantly enhanced at the operating excitation wavelength thanks to efficient exciton funneling from the CdS crown, enabling ultralow threshold population inversion condition. Furthermore, thanks to the larger lateral size of the multicrown heteronanoplatelets compared to the conventional type-II NPLs, Auger recombination is further suppressed, resulting in a significant reduction of the threshold for the ASE. 
Figure $4 \mathrm{~b}$ shows the integrated $\mathrm{PL}$ intensity of the multicrown $\mathrm{CdSe} / \mathrm{CdSe}_{1-x} \mathrm{Te}_{x} / \mathrm{CdS} \quad(\mathrm{x}=0.25,0.40,0.50)$ type-II NPLs as a function of the 1PA pumping intensity. The lowest stimulated emission threshold is found to be as low as $4.15 \mu \mathrm{J} / \mathrm{cm}^{2}$ for $\mathrm{CdSe} / \mathrm{CdSe} e_{0.75} \mathrm{Te}_{0.25} / \mathrm{CdS}$ type-II NPLs. Also, we investigated different batches of the CdSe/ $\mathrm{CdSe}_{0.75} \mathrm{Te}_{0.25} / \mathrm{CdS}$ NPLs, which exhibited ASE thresholds in the range of $4-7 \mu \mathrm{J} / \mathrm{cm}^{2}$. Moreover, other compositions of the $\mathrm{CdSe} / \mathrm{CdSe}_{1-x} \mathrm{Te}_{x} / \mathrm{CdS}$ type-II NPLs led to the ASE thresholds of 18.45 and $12.48 \mu \mathrm{J} / \mathrm{cm}^{2}$ for $x=0.40$ and 0.50 , respectively. The lowest obtained 1PA-pumped ASE thresholds $\left(I_{\text {th }}\right)$ are comparable to or better than the recently reported high-performance engineered heterostructures of the colloidal semiconductor nanocrystals including QDs (for $\mathrm{CdSe} / \mathrm{CdZnS}$ core/shell QDs, $I_{\mathrm{th}} \cong 90 \mu \mathrm{J} / \mathrm{cm}^{2} ;{ }^{39}$ and for CdSe/CdS giant-shell QDs, $I_{\mathrm{th}} \cong 29 \mu \mathrm{J} / \mathrm{cm}^{240,41}$ and $I_{\mathrm{th}} \cong 6 \mu \mathrm{J} /$ $\mathrm{cm}^{242}$ ), perovskites (for organic-inorganic halide perovskites, $I_{\text {th }} \cong 10 \pm 2 \mu \mathrm{J} / \mathrm{cm}^{2} ; 4$ and for cesium lead halide perovskites, $I_{\mathrm{th}} \cong 5 \pm 1 \mu \mathrm{J} / \mathrm{cm}^{244}$ ) and NPLs (for CdSe/CdS core/crown type-I NPLs, $I_{\text {th }} \cong 41 \mu \mathrm{J} / \mathrm{cm}^{2} ;{ }^{6}$ for CdSe/CdS core/shell quasitype-II NPLs, $I_{\text {th }} \cong 6 \mu \mathrm{J} / \mathrm{cm}^{2} ;{ }^{45}$ for CdSe/CdS@CdS core/ crown@shell NPLs, $I_{\mathrm{th}} \cong 23 \mu \mathrm{J} / \mathrm{cm}^{2}$; $^{21}$ and for CdSe/ $\mathrm{CdSe}_{1-x} \mathrm{Te}_{x}$ core/alloyed-crown type-II NPLs, $I_{\mathrm{th}} \cong 26 \mu \mathrm{J} /$ $\mathrm{cm}^{229}$ ). Therefore, multicrown type-II heteronanoplatelets demonstrate highly efficient optical gain performance owing to a large absorption cross section, highly suppressed Auger recombination, and strong optical absorption at the pump laser wavelength thanks to the growth of the CdS crown around the alloyed-crown type-II NPLs.

Moreover, we studied the 2PA-pumped $\left(\lambda_{\text {exc }}=800 \mathrm{~nm}\right)$ optical gain performance of type-II NPLs in CdSe/ $\mathrm{CdSe}_{0.75} \mathrm{Te}_{0.25} / \mathrm{CdS}$ NPLs. The type-II like electronic structure and growth of the CdS crown around the alloyed-crown make these NPLs promising candidates for low-threshold-frequency up-converted lasers owing to their large nonlinear absorption cross section. The emission spectra of 2PA-pumped ASE are presented as a function of pumping fluence in Figure 4c. Similar to 1PA, the ASE peak is $\sim 3 \mathrm{~nm}$ blue-shifted compared to the spontaneous emission peak. Figure $4 \mathrm{~d}$ presents the integrated $\mathrm{PL}$ intensity of the multicrown $\mathrm{CdSe} /$ $\mathrm{CdSe}_{0.75} \mathrm{Te}_{0.25} / \mathrm{CdS}$ type-II NPLs versus the pump fluence. The 2PA-pumped ASE threshold is $2.48 \mathrm{~mJ} / \mathrm{cm}^{2}$, which is the lowest reported to date among all colloidal semiconductor nanocrystals, to the best of our knowledge.

To better understand the optical gain performance of the samples, we investigated the net peak modal gain $(G)$ of the multicrown type-II NPLs (thin-film samples), which is a critical parameter for a gain medium in light amplification. $G$ assesses the amount of light amplification per unit length and depends on the material gain coefficient and total optical loss coefficient of the medium (i.e., waveguide loss coefficient). We characterized the net modal coefficient via variable stripe length (VSL) measurement (see the Supporting Information for details). Figure 5a represents the VSL measurements of the $\mathrm{CdSe} / \mathrm{CdSe}_{0.75} \mathrm{Te}_{0.25} / \mathrm{CdS}$ multicrown type-II NPLs at the ASE peak as a function of pump fluences. To calculate the net peak modal gain $(G)$, we employed the following expression ${ }^{46}$

$$
I(l)=\frac{I_{\mathrm{sp}}}{G}\left(\mathrm{e}^{G l}-1\right)
$$

where $I(l)$ is the ASE peak intensity, $I_{\text {sp }}$ is a constant proportional to the spontaneous emission, $G$ is the net peak modal gain coefficient, and $l$ shows the length of the stripe in the VSL measurements. The experimental VSL data are fitted by eq 1 to extract the net peak modal gain $(G)$. Then, $G$ is plotted as a function of the excitation intensity in Figure $5 b$, to further investigate the optical gain performance of the $\mathrm{CdSe} /$ $\mathrm{CdSe}_{0.75} \mathrm{Te}_{0.25} / \mathrm{CdS}$ multicrown type-II NPLs, we fitted the calculated net peak modal gain by using the following relation $^{29,47}$

$$
G=g-\alpha=\Gamma \times \sigma \times \frac{\phi_{\mathrm{p}}}{1+\frac{\phi_{\mathrm{p}}}{\phi_{\mathrm{s}}}}
$$

where $G$ is the net peak modal gain, $g$ is the peak modal gain, and $\alpha$ presents the total loss of the system. $\Gamma$ is the optical mode confinement in the closely packed film, $\sigma$ is the absorption cross section of the NPLs in the stimulated emission, $\phi_{\mathrm{p}}$ represents the pump intensity, and $\phi_{\mathrm{s}}$ is the saturation pump fluence. As can be seen in Figure $5 \mathrm{~b}$, the net peak modal gain depicts a linear behavior at the low pump intensity, and then is followed by a gradual saturation behavior at the elevated excitation fluence. From numerical fitting of the experimental data by eq 2 , the net peak modal gain coefficient was found to be as high as $520 \pm 32 \mathrm{~cm}^{-1}$ at $286 \mu \mathrm{J} / \mathrm{cm}^{2}$ while $\phi_{\mathrm{s}}$ is $\sim 360 \mu \mathrm{J} / \mathrm{cm}^{2}$. Our attained net peak modal gain is comparable to or better than the previously reported net modal gains for the heterostructure of colloidal semiconductor nanocrystals including $\mathrm{CdSe} / \mathrm{ZnCdS}$ core/shell QDs $(G=$ $\left.95 \pm 10 \mathrm{~cm}^{-1}\right)$ and organic-inorganic halide perovskite $(\mathrm{G} \cong$ $\left.250 \mathrm{~cm}^{-1}\right) .{ }^{43}$ However, Guzelturk et al. ${ }^{29}$ reported a net modal gain as high as $\sim 930 \mathrm{~cm}^{-1}$ for the alloyed-crown type-II NPLs, which can be attributed to the face-to-face stacking of these NPLs in their solid films, making them strongly closely packed assembled films. Due to stacking of NPLs, however, the scattering is also increased in their solid films, resulting in a relatively high waveguide loss coefficient $\left(\sim 46 \mathrm{~cm}^{-1}\right)$.

Figure 5c depicts the ASE peak intensity as a function of distance from the edge of the sample by using a simple numerical fitting $\left(I(l)=I_{0} \mathrm{e}^{-\alpha l}\right.$, where $\alpha$ represents the waveguide loss coefficient and $l$ is the distance from edge of the thin film). Here, $\alpha$ was obtained to be as low as $18 \mathrm{~cm}^{-1}$ for the spin-coated solid film of the multicrown type-II NPLs, which is the lowest optical loss coefficient ( $\sim 3$-fold smaller) reported in the colloidal semiconductor nanocrystals including $\mathrm{QDs}^{48}$ and NPLs ${ }^{29}$ to date (see the Supporting Information for details). We ascribed this very low optical loss of the system to the multicrown structure of the engineered NPLs, which enables us to significantly reduce the face-to-face stacking of the NPLs in the closely packed solid films resulting in the decreased scattering. Then, the peak modal gain $(g)$ is plotted versus the excitation fluence in Figure $5 d$, where the optical gain occurs when the modal gain coefficient beats the total loss of the system. As a result, we realized an optical transparency obtained at $\sim 4 \mu \mathrm{J} / \mathrm{cm}^{2}$ excitation intensity, which is fully consistent with our achieved ASE threshold for $x=0.25$ multicrown type-II NPLs. We attribute this low threshold of the optical gain to the significant reduction of the system losses (i.e., waveguide loss coefficient), which was previously predicted by Guzelturk et al. ${ }^{29}$

In conclusion, due to the poor colloidal stability under ambient conditions and great tendency to form long needlelike stacks, four-ML-thick $\mathrm{CdSe} / \mathrm{CdSe}_{1-x} \mathrm{Te}_{x}$ core/alloyedcrown type-II NPLs are limited to exploit their potentially exceptional optical performance. By growing a CdS crown 
around their periphery, we enhanced both colloidal and photostability of the multicrown $\mathrm{CdSe} / \mathrm{CdSe}_{1-x} \mathrm{Te}_{x} / \mathrm{CdS}$ core/ alloyed-crown/crown heterostructure NPLs having Te amount $\leq 0.50$. By using the proposed multicrown heterostructures as a gain medium, we obtained the lowest gain thresholds under 1PA- and 2PA-pumping conditions. We attributed these significantly reduced gain thresholds in these engineered heterostructured NPLs to their enhanced stability and reduced tendency to form stacks. All these findings indicate that having highly stable multicrown structure make these nanoplatelets an attractive choice for next-generation optoelectronic devices.

\section{ASSOCIATED CONTENT}

\section{S Supporting Information}

The Supporting Information is available free of charge on the ACS Publications website at DOI: 10.1021/acs.chemmater.9b00136.

Four-ML-thick CdSe core characterization, analysis of high-resolution XPS spectra of NPLs, HAADF-STEM images of $\mathrm{CdSe} / \mathrm{CdSe}_{1-x} \mathrm{Te}_{x}$ and their $\mathrm{CdSe} /$ $\mathrm{CdSe}_{1-x} \mathrm{Te}_{x} / \mathrm{CdS}$ multicrown structures for different $x$ values, photoluminescence (PL) and photoluminescence excitation (PLE) spectra of $\mathrm{CdSe} / \mathrm{CdSe}_{1-x} \mathrm{Te}_{x} / \mathrm{CdS}$ structures, analysis of time-resolved fluorescence decay curves, amplified spontaneous emission (ASE) spectra of multicrown type-II NPLs, schematic demonstration of multicrown type-II colloidal quantum wells, and experimental details of variable stripe length (VSL) and waveguide loss coefficient measurements (PDF)

\section{AUTHOR INFORMATION}

\section{Corresponding Author}

*E-mail: volkan@stanfordalumni.org; volkan@bilkent.edu.tr; hvdemir@ntu.edu.sg.

\section{Author Contributions}

${ }^{\#}$ D.D. and N.T. contributed equally.

Notes

The authors declare no competing financial interest.

\section{ACKNOWLEDGMENTS}

The authors gratefully acknowledge the financial support in part from the Singapore National Research Foundation under the programs of NRF-NRFI2016-08 and the Science and Engineering Research Council, Agency for Science, Technology and Research (A*STAR) of Singapore and in part from TUBITAK 114F326 and 115E679. H.V.D. also acknowledges support from TUBA. K.G. and Y.K. acknowledge support from TUBITAK BIDEB.

\section{REFERENCES}

(1) Talapin, D. V.; Lee, J.-S.; Kovalenko, M. V.; Shevchenko, E. V. Prospects of Colloidal Nanocrystals for Electronic and Optoelectronic Applications. Chem. Rev. 2010, 110, 389-458.

(2) Pietryga, J. M.; Park, Y.-S.; Lim, J.; Fidler, A. F.; Bae, W. K.; Brovelli, S.; Klimov, V. I. Spectroscopic and Device Aspects of Nanocrystal Quantum Dots. Chem. Rev. 2016, 116, 10513-10622.

(3) Ithurria, S.; Dubertret, B. Quasi 2D Colloidal CdSe Platelets with Thicknesses Controlled at the Atomic Level. J. Am. Chem. Soc. 2008, 130, 16504-16505.

(4) Nasilowski, M.; Mahler, B.; Lhuillier, E.; Ithurria, S.; Dubertret, B. Two-Dimensional Colloidal Nanocrystals. Chem. Rev. 2016, 116, 10934-10982.
(5) Pelton, M. Carrier Dynamics, Optical Gain, and Lasing with Colloidal Quantum Wells. J. Phys. Chem. C 2018, 122, 10659-10674.

(6) Guzelturk, B.; Kelestemur, Y.; Olutas, M.; Delikanli, S.; Demir, H. V. Amplified Spontaneous Emission and Lasing in Colloidal Nanoplatelets. ACS Nano 2014, 8, 6599-6605.

(7) Ithurria, S.; Tessier, M. D.; Mahler, B.; Lobo, R. P. S. M.; Dubertret, B.; Efros, A. L. Colloidal Nanoplatelets with TwoDimensional Electronic Structure. Nat. Mater. 2011, 10, 936-941.

(8) Olutas, M.; Guzelturk, B.; Kelestemur, Y.; Yeltik, A.; Delikanli, S.; Demir, H. V. Lateral Size-Dependent Spontaneous and Stimulated Emission Properties in Colloidal CdSe Nanoplatelets. ACS Nano 2015, 9, 5041-5050.

(9) Li, Q.; Lian, T. A Model for Optical Gain in Colloidal Nanoplatelets. Chem. Sci. 2018, 9, 728-734.

(10) Kelestemur, Y.; Dede, D.; Gungor, K.; Usanmaz, C. F.; Erdem, O.; Demir, H. V. Alloyed Heterostructures of CdSexS1-x Nanoplatelets with Highly Tunable Optical Gain Performance. Chem. Mater. 2017, 29, 4857-4865.

(11) She, C.; Fedin, I.; Dolzhnikov, D. S.; Dahlberg, P. D.; Engel, G. S.; Schaller, R. D.; Talapin, D. V. Red, Yellow, Green, and Blue Amplified Spontaneous Emission and Lasing Using Colloidal CdSe Nanoplatelets. ACS Nano 2015, 9, 9475-9485.

(12) Li, M.; Zhi, M.; Zhu, H.; Wu, W.-Y.; Xu, Q.-H.; Jhon, M. H.; Chan, Y. Ultralow-Threshold Multiphoton-Pumped Lasing from Colloidal Nanoplatelets in Solution. Nat. Commun. 2015, 6, 8513.

(13) Giovanella, U.; Pasini, M.; Lorenzon, M.; Galeotti, F.; Lucchi, C.; Meinardi, F.; Luzzati, S.; Dubertret, B.; Brovelli, S. Efficient Solution-Processed Nanoplatelet-Based Light-Emitting Diodes with High Operational Stability in Air. Nano Lett. 2018, 18, 3441-3448.

(14) Fan, F.; Kanjanaboos, P.; Saravanapavanantham, M.; Beauregard, E.; Ingram, G.; Yassitepe, E.; Adachi, M. M.; Voznyy, O.; Johnston, A. K.; Walters, G.; et al. Colloidal CdSe1-xSx Nanoplatelets with Narrow and Continuously- Tunable Electroluminescence. Nano Lett. 2015, 15, 4611-4615.

(15) Erdem, T.; Demir, H. V. Colloidal Nanocrystals for Quality Lighting and Displays : Milestones and Recent Developments. Nanophotonics 2016, 5, 74-95.

(16) Taghipour, N.; Ludwig, P.; Martinez, H.; Ozden, A.; Olutas, M.; Dede, D.; Gungor, K.; Erdem, O.; Perkgoz, N. K.; Demir, H. V. Near-Unity Efficiency Energy Transfer from Colloidal Semiconductor Quantum Wells of CdSe/CdS Nanoplatelets to a Monolayer of MoS2. ACS Nano 2018, 12, 8547-8554.

(17) Guzelturk, B.; Olutas, M.; Delikanli, S.; Kelestemur, Y.; Erdem, O.; Demir, H. V. Nanoscale Nonradiative Energy Transfer in Colloidal CdSe. Nanoscale 2015, 7, 2545-2551.

(18) Sharma, M.; Gungor, K.; Yeltik, A.; Olutas, M.; Guzelturk, B.; Kelestemur, Y.; Erdem, T.; Delikanli, S.; McBride, J. R.; Demir, H. V. Near-Unity Emitting Copper-Doped Colloidal Semiconductor Quantum Wells for Luminescent Solar Concentrators. Adv. Mater. 2017, 29, 1-10.

(19) Tessier, M. D.; Spinicelli, P.; Dupont, D.; Patriarche, G.; Ithurria, S.; Dubertret, B. Efficient Exciton Concentrators Built from Colloidal Core/Crown CdSe/CdS Semiconductor Nanoplatelets. Nano Lett. 2014, 14, 207-213.

(20) Ithurria, S.; Talapin, D. V. Colloidal Atomic Layer Deposition (c-ALD) Using Self-Limiting Reactions at Nanocrystal Surface Coupled to Phase Transfer between Polar and Nonpolar Media. J. Am. Chem. Soc. 2012, 134, 18585-18590.

(21) Kelestemur, Y.; Guzelturk, B.; Erdem, O.; Olutas, M.; Gungor, K.; Demir, H. V. Platelet-in-Box Colloidal Quantum Wells: CdSe/ CdS@CdS Core/Crown@Shell Heteronanoplatelets. Adv. Funct. Mater. 2016, 26, 3570-3579.

(22) Li, Q.; Wu, K.; Chen, J.; Chen, Z.; McBride, J. R.; Lian, T. SizeIndependent Exciton Localization Efficiency in Colloidal CdSe/CdS Core/Crown Nanosheet Type-I Heterostructures. ACS Nano 2016, 10, 3843-3851.

(23) Delikanli, S.; Guzelturk, B.; Hernández-Martínez, P. L.; Erdem, T.; Kelestemur, Y.; Olutas, M.; Akgul, M. Z.; Demir, H. V. Continuously Tunable Emission in Inverted Type-I CdS/CdSe 
Core/Crown Semiconductor Nanoplatelets. Adv. Funct. Mater. 2015, $25,4282-4289$.

(24) Kelestemur, Y.; Olutas, M.; Delikanli, S.; Guzelturk, B.; Akgul, M. Z.; Demir, H. V. Type-II Colloidal Quantum Wells: CdSe/CdTe Core/Crown Heteronanoplatelets. J. Phys. Chem. C 2015, 119, 21772185.

(25) Pedetti, S.; Ithurria, S.; Heuclin, H.; Patriarche, G.; Dubertret, B. Type-II CdSe/CdTe Core/Crown Semiconductor Nanoplatelets. J. Am. Chem. Soc. 2014, 136, 16430-16438.

(26) Antanovich, A. V.; Prudnikau, A. V.; Melnikau, D.; Rakovich, Y. P.; Chuvilin, A.; Woggon, U.; Achtstein, A. W.; Artemyev, M. V. Colloidal Synthesis and Optical Properties of Type-II CdSe-CdTe and Inverted CdTe-CdSe Core-Wing Heteronanoplatelets. Nanoscale 2015, 7, 8084-8092.

(27) Kelestemur, Y.; Guzelturk, B.; Erdem, O.; Olutas, M.; Erdem, T.; Usanmaz, C. F.; Gungor, K.; Demir, H. V. CdSe/CdSe1XTexCore/Crown Heteronanoplatelets: Tuning the Excitonic Properties without Changing the Thickness. J. Phys. Chem. C 2017, 121, $4650-4658$.

(28) Dufour, M.; Steinmetz, V.; Izquierdo, E.; Pons, T.; Lequeux, N.; Lhuillier, E.; Legrand, L.; Chamarro, M.; Barisien, T.; Ithurria, S. Engineering Bicolor Emission in 2D Core/Crown CdSe/CdSe1-XTex Nanoplatelet Heterostructures Using Band-Offset Tuning. J. Phys. Chem. C 2017, 121, 24816-24823.

(29) Guzelturk, B.; Kelestemur, Y.; Olutas, M.; Li, Q.; Lian, T.; Demir, H. V. High-Efficiency Optical Gain in Type-II Semiconductor Nanocrystals of Alloyed Colloidal Quantum Wells. J. Phys. Chem. Lett. 2017, 8, 5317-5324.

(30) Li, Q.; Xu, Z.; McBride, J. R.; Lian, T. Low Threshold Multiexciton Optical Gain in Colloidal CdSe/CdTe Core/Crown Type-II Nanoplatelet Heterostructures. ACS Nano 2017, 11, 25452553.

(31) Liu, B.; Delikanli, S.; Gao, Y.; Dede, D.; Gungor, K.; Demir, H. V. Nanocrystal Light-Emitting Diodes Based on Type II Nanoplatelets. Nano Energy 2018, 47, 115-122.

(32) de Mello, J. C.; Wittmann, H. F.; Friend, R. H. An Improved Experimental Determination of External Photoluminescence Quantum Efficiency. Adv. Mater. 1997, 9, 230-232.

(33) Jana, S.; De Frutos, M.; Davidson, P.; Abécassis, B. LigandInduced Twisting of Nanoplatelets and Their Self-Assembly into Chiral Ribbons. Sci. Adv. 2017, 3, No. e1701483.

(34) Gao, Y.; Li, M.; Delikanli, S.; Zheng, H.; Liu, B.; Dang, C.; Sum, T. C.; Demir, H. V. Low-Threshold Lasing from Colloidal CdSe/CdSeTe Core/Alloyed-Crown Type-II Heteronanoplatelets. Nanoscale 2018, 10, 9466-9475.

(35) Wei, S. H.; Zhang, S. B. Structure Stability and Carrier Localization in $\mathrm{CdX}(\mathrm{X}=\mathrm{S}, \mathrm{Se}, \mathrm{Te})$ Semiconductors. Phys. Rev. B: Condens. Matter Mater. Phys. 2000, 62, 6944-6947.

(36) Cihan, A. F.; Kelestemur, Y.; Guzelturk, B.; Yerli, O.; Kurum, U.; Yaglioglu, H. G.; Elmali, A.; Demir, H. V. Attractive versus Repulsive Excitonic Interactions of Colloidal Quantum Dots Control Blue- to Red-Shifting (and Non-Shifting) Amplified Spontaneous Emission. J. Phys. Chem. Lett. 2013, 4, 4146-4152.

(37) Cassette, E.; Pedetti, S.; Mahler, B.; Ithurria, S.; Dubertret, B.; Scholes, G. D. Ultrafast Exciton Dynamics in 2D In-Plane HeteroNanostructures: Delocalization and Charge Transfer. Phys. Chem. Chem. Phys. 2017, 8373-8379.

(38) Kelestemur, Y.; Cihan, A. F.; Guzelturk, B.; Demir, H. V. TypeTunable Amplified Spontaneous Emission from Core-Seeded CdSe/ CdS Nanorods Controlled by Exciton-Exciton Interaction. Nanoscale 2014, 6, 8509-8514.

(39) Dang, C.; Lee, J.; Breen, C.; Steckel, J. S.; Coe-Sullivan, S.; Nurmikko, A. Red, Green and Blue Lasing Enabled by Single-Exciton Gain in Colloidal Quantum Dot Films. Nat. Nanotechnol. 2012, 7, 335-339.

(40) García-Santamaría, F.; Chen, Y.; Vela, J.; Schaller, R. D.; Hollingsworth, J. A.; Klimov, V. I. Suppressed Auger Recombination in "Giant” Nanocrystals Boosts Optical Gain Performance. Nano Lett. 2009, 9, 3482-3488.
(41) Guzelturk, B.; Kelestemur, Y.; Gungor, K.; Yeltik, A.; Akgul, M. Z.; Wang, Y.; Chen, R.; Dang, C.; Sun, H.; Demir, H. V. Stable and Low-Threshold Optical Gain in CdSe/CdS Quantum Dots: An AllColloidal Frequency up-Converted Laser. Adv. Mater. 2015, 27, 2741-2746.

(42) Park, Y.-S.; Bae, W. K.; Baker, T.; Lim, J.; Klimov, V. I. Effect of Auger Recombination on Lasing in Heterostructured Quantum Dots with Engineered Core/Shell Interfaces. Nano Lett. 2015, 15, 73197328 .

(43) Xing, G.; Mathews, N.; Lim, S. S.; Yantara, N.; Liu, X.; Sabba, D.; Grätzel, M.; Mhaisalkar, S.; Sum, T. C. Low-Temperature Solution-Processed Wavelength-Tunable Perovskites for Lasing. Nat. Mater. 2014, 13, 476-480.

(44) Yakunin, S.; Protesescu, L.; Krieg, F.; Bodnarchuk, M. I.; Nedelcu, G.; Humer, M.; De Luca, G.; Fiebig, M.; Heiss, W.; Kovalenko, M. V. Low-Threshold Amplified Spontaneous Emission and Lasing from Colloidal Nanocrystals of Caesium Lead Halide Perovskites. Nat. Commun. 2015, 6, 8056.

(45) She, C.; Fedin, I.; Dolzhnikov, D. S.; Demortière, A.; Schaller, R. D.; Pelton, M.; Talapin, D. V. Low-Threshold Stimulated Emission Using Colloidal Quantum Wells. Nano Lett. 2014, 14, 2772-2777.

(46) Samuel, I. D. W.; Turnbull, G. A. Organic Semiconductor Lasers. Chem. Rev. 2007, 107, 1272-1295.

(47) Koechner, W. Solid-State Laser Engineering, 6th ed.; SpringerVerlag New York, 2006.

(48) Lin, C. H.; Lafalce, E.; Jung, J.; Smith, M. J.; Malak, S. T.; Aryal, S.; Yoon, Y. J.; Zhai, Y.; Lin, Z.; Vardeny, Z. V.; et al. Core/AlloyedShell Quantum Dot Robust Solid Films with High Optical Gains. ACS Photonics 2016, 3, 647-658. 